\title{
Ontologies and Representation Spaces for Sketch Map Interpretation
}

\author{
Jia Wang ${ }^{*}$ and Michael Worboys \\ Department of Computing \& Information Systems, University of Greenwich, London, \\ United Kingdom
}

\begin{abstract}
In this paper, we present a systematic approach to sketch map interpretation. The method decomposes the elements of a sketch map into a hierarchy of categories, from the material sketch map level to the non-material representational sketch map level, and then interprets the sketch map using the five formal representation spaces that we develop. These spaces (set, graph, metric and Euclidean) provide a tiered formal representation based on standard mathematical structures. We take the view that a sketch map bears information about the physical world and systematises this using extensions of existing formal ontologies. The motivation for this work is the partially automatic extraction and integration of information from sketch maps. We propose a set of ontologies and methods as a first step in the direction of a formalisation of partially automatic extraction and integration of sketch map content. We also see this work as a contribution to spatial cognition, where researchers externalise spatial knowledge using sketch mapping. The paper concludes by working through an example that demonstrates the sketch map interpretation at different levels using the underlying method.
\end{abstract}

Keywords: sketch map; interpretation; representation space; ontology; information artefact

\section{Introduction}

Sketch maps are drawn by people as a way to communicate information about locations and routes. They are produced deliberately about some portion of reality that is perceived and stored as cognitive representations in memory. Such representations are called cognitive maps (Tolman 1948). Information stored in sketch maps can be used to infer human spatial knowledge represented in cognitive maps. As Billinghurst and Weghorst (1995, p. 40) wrote, "the fundamental importance of an effective cognitive map is that it allows two questions to be answered quickly and efficiently: where is that? how do I get to there from here?" Sketch maps as an externalisation of cognitive maps are a valid measurement of such cognition information (Newcombe 1985, Blades 1990). As information artefacts, sketch maps exist independently of their cognitive counterparts and transform such mental representations to make them publicly observable and inspectable. Figure 1 shows the connections between sketch maps, cognitive maps, spatial knowledge and the portions of reality sketch maps intend to depict. Cognitive maps serve the construction and accumulation of spatial knowledge obtained in reality through the mind's eye (one's ability to 'see' things using sensory organs with the mind). Sketch maps partially project cognitive maps and reflect acquired spatial knowledge on to paper or other media outside the human mind so that we are able to infer spatial knowledge from sketch map information.

\footnotetext{
*Corresponding author. Email: J.Wang@greenwich.ac.uk
} 


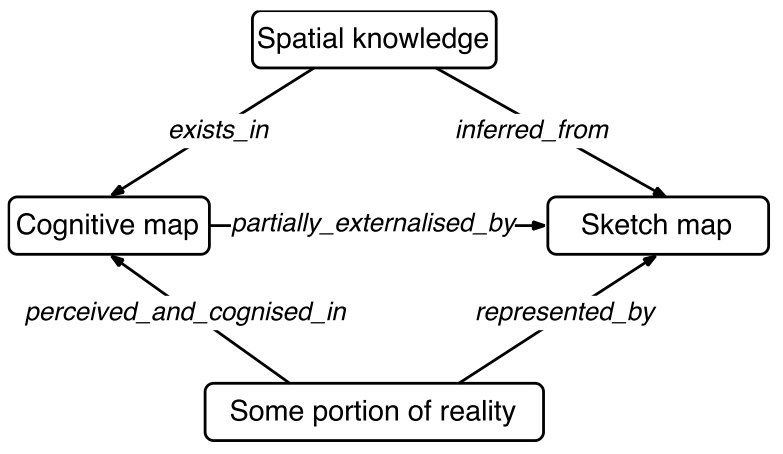

Figure 1. Connections between spatial knowledge, cognitive maps, sketch maps and reality

We need methods that classify and quantify (or qualify) features, especially spatial features of sketch maps, in order to obtain the spatial knowledge stored in cognitive maps. Such approaches are determined by the interpretation of the types of information that can be extracted from sketch maps. Compared to the intensive use of sketch maps to evaluate cognitive maps as early as Lynch (1960) (cf. Appleyard 1970, Lloyd and Heivly 1987, Tversky 1999, Ishikawa 2008) and the interest in cognition information (e.g. Kuipers 1983), less work has been done on sketch map interpretation for cognitive map measurement.

In this study, sketch map interpretation is defined as finding ways of structuring and representing the spatial information that can be extracted from sketch maps. This definition is different from most existing studies in computer vision that treat interpretation as a matter of recognising ink and aim at translating strokes and shapes into object descriptions (Davis 2002, 2007). Different from open-domain sketch interpretation in CogSketch (Forbus et al. 2011), our study is restricted and based on two assumptions: first, our working domain is limited to the sketch maps of urban areas from a survey perspective with a well-established graphic lexicon and grammar; second, our application domain is restricted to geographic information science and spatial cognition where sketch maps are used mainly for spatial knowledge externalisation. According to Davis (2002), a graphic lexicon is similar to a vocabulary in language which defines the set of shapes used in a domain. For example, artificial objects such as buildings are usually drawn as regular shapes while geographical objects such as water bodies are drawn as irregular blob shapes (Wang 2014). The grammar describes the interrelations among these shapes to indicate spatial relations such as adjacency or containment. In this paper, we define a sketch map as a freehand physical drawing made on a piece of paper, and such a drawing is from memory. Also, the sketch map is about an urban area at environmental scale (Montello 1993). Note that sketch maps do not have to be map-like representations even though the term 'sketch map' has the word 'map' in it.

We think consistent and widely accepted ontological resources (including basic taxonomies as well as more complex ontologies) are necessary for sketch map interpretation. The semantic interpretation of sketch maps can benefit from the definitions of useful concepts and the links between them expressed formally as ontologies. We propose a formal method to interpret sketch maps using a hierarchy of ontological levels, each populated by its distinctive entities and related in systematic ways to entities at other levels. This method allows us to interpret and model sketch information using several formal representation spaces, such as graph and metric spaces, where existing computational approaches are available for spatial analyses in 
each representation space and transformations between different spaces. A set of ontologies of sketch maps is introduced as a first step in the direction of a formalisation of partially automatic extraction and integration of sketch map content. The introduced ontologies demonstrate first how non-material sketch entities (sketch information) at different levels are embedded in corresponding material sketch entities (physical sketch maps), and second how we can infer spatial information from these non-material entities. In the final sections, we present an illustrative example to demonstrate our method and conclude the study.

Our work provides consistent ontological resources and a formal model used for sketch map interpretation for researchers who want to retrieve and analyse sketch information for evaluating cognitive maps and acquired human spatial knowledge, and where possibly we draw links between our work and existing ontologies based on the Basic Formal Ontology (BFO) such as the Information Artefact Ontology (IAO) (Smith et al. 2013), as well as work from the computer vision domain.

Moreover, our work contributes to automatic sketch information retrieval used by those sketch map based systems such as SketchMapia proposed by Schwering et al. (2014). The contribution is twofold. At the front end, our formal model provides types, properties and interrelations of relevant sketch features to be recognised by a sketching interface. At the back end, our ontologies and taxonomies help to design a sketch database schema with consistent structures and constraints on both sketch map data and spatial query.

\section{Background}

We review related work in three areas: sketch mapping for cognitive map evaluation, existing approaches to sketch understanding and interpretation, and ontologies of information artefacts.

Sketch maps have been used as the main approach to the elicitation of cognitive maps. Lynch (1960) used sketch maps to study how people internally represent their local cities. Tversky (1999) found it was sufficient to employ sketch maps together with a direction toolkit to convey routes. The central interests to use sketch maps to assess cognitive maps include studying distortions, schematisation and other inaccuracies by comparing sketch objects and ground objects (Lloyd and Heivly 1987), understanding how people form cognitive maps and what information cognitive maps convey (Appleyard 1970, Kuipers 1983, Chrastil and Warren 2014), and studying the invariant sketch map information and applying it to a computational environment for querying spatial databases (Wang and Schwering 2015).

Scientists from the computer vision domain are also interested in sketch maps (or, more generally, sketches) but have their focus on computer understanding of sketches in a manner similar to a human observer (Davis 2007). Different from formal diagrams, sketches contain noise such as unintended overlapping shapes and gaps in circles. Sketch interpretation in this domain attempts to recognise the shape of the object drawn using the same notion of shape that people use. For online sketches, the direction, curvature and speed of drawing are recorded, and a sketch at its bottom level is a collection of strokes, each of which is a set of time-stamped coordinates. Shapes at a higher level are recognised by observing stroke sequences and matching them locally against templates using metric distances or shape contexts (Davis 2007). It is worth mentioning the online sketch understanding platform, CogSketch, made for cognitive science research and education. Using analogical reasoning, CogSketch combines its visual, spatial and conceptual knowledge about sketch elements to create a qualitative, 
symbolic representation both of the sketch and of what it depicts (Forbus et al. 2011). Sketches in CogSketch are separated into multiple sub-sketches, with each sub-sketch consisting of one or more layers. Each layer has a genre and pose, both of which construct spatial relationships. For offline sketches (e.g. scanned paper-based sketches), sketch images can be first segmented as a collection of independent sketch objects and then refined using local context-aware recognition (Broelemann et al. 2016). Other methods of detecting sketch shapes include using heuristic rules with a predefined and restricted set of elements (Chen and Takagi 2013).

Sketch interpretation can benefit from consistent and widely accepted ontological resources, which provide general taxonomies of what exists in a sketch map as well as complex relationships between sketch information entities. Some of the most commonly used upper ontologies are the Basic Formal Ontology (BFO), the Descriptive Ontology for Linguistic and Cognitive Engineering (DOLCE) (Gangemi et al. 2002) and the Suggested Upper Merged Ontology (SUMO). BFO-based ontologies can incorporate both continuant and occurrent entities in a single framework to represent a portion of reality at a given level of granularity (Arp et al. 2015). SUMO was developed by the IEEE (Niles and Pease 2001), and its mapping to WordNet has made it used frequently for natural language processing tasks.

In this paper, the choice of a BFO-based approach lies in the fact that BFO has the well-established IAO (Information Artefact Ontology), which can represent quality, structure and content of information in a sketch map. IAO ${ }^{1}$ is an ontology of information entities that uses BFO as its foundational ontology (Arp et al. 2015). An information artefact is a material entity whose function is to bear an information quality entity (Smith et al. 2013). When we talk about an information artefact, we refer to both a continuant physical artefact such as a physical paper-based sketch map as an information bearing entity and a continuant information entity carried by the physical artefact (Smith and Malyuta 2015). IAO contains several fundamental information entities that describe quality, content and structure of an information artefact (such as a sketch map). The core of IAO contains a small number of foundational information entities that include both representative and non-representative aspects of an information artefact. Figure 2 provides the IAO framework based on BFO entities (in black). The representative aspect (in blue) refers to an information content entity (ICE), which is a generically dependent continuant fixed in an Information Bearing Entity (IBE) and intended to refer to some entities external to the representation. The nonrepresentative aspects (in red) include the IBE, information quality entity (IQE) and information structure entity (ISE). An IQE is the pattern on an IBE by virtue of which it is a bearer of some information entity, and an ISE is the structural part of an ICE. This framework was reused by Galton et al. (2016) to develop a set of ontologies to interpret images of cell and tissues.

\footnotetext{
${ }^{1}$ http://bioportal.bioontology.org/ontologies/IAO
} 


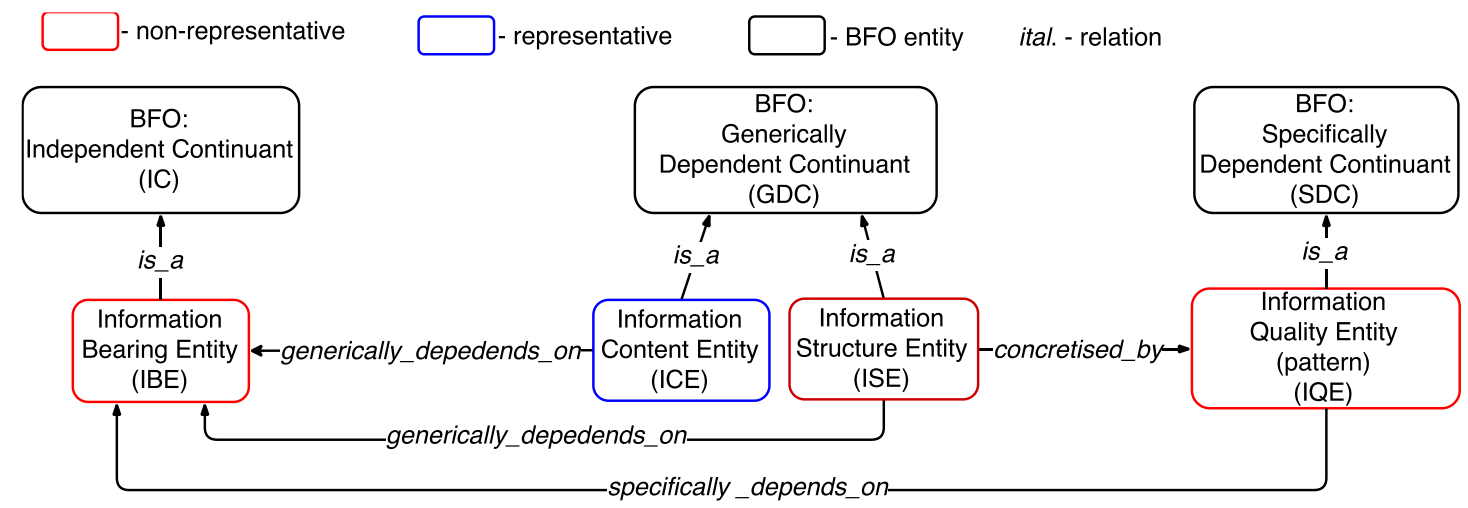

Figure 2. IAO Framework (Smith et al. 2013)

Galton and Worboys (2011) proposed an ontology of information, whose basic framework is reused in our study to represent interrelations between different information entities.

\section{Levels of Sketch Map Interpretation}

It is helpful to think of our approach in terms of a number of different levels, representing the stages in deriving sketch elements of different types from the bottom material sketch map level to the higher non-material representational sketch map level. Figure 3 illustrates this hierarchy of sketch map interpretation. At the material sketch map level (in black), we have paper-based sketch maps as bearers for non-material sketch information. At the representational sketch map level (in blue), we have two types of representation, namely sketch image and real-world. Both representations exist in physical sketch maps and are supported by representation primitives. The sketch image representation is only concerned with the visual elements and the composition of these elements in a sketch map, while the real-world representation makes use of domain-specific knowledge about the real-world objects being depicted. The real-world representation is usually indicated by the sketch image representation. Following the IAO definitions (Figure 2), a material sketch map is an information bearing entity, and both representations as well as the representation primitives are information content entities. 


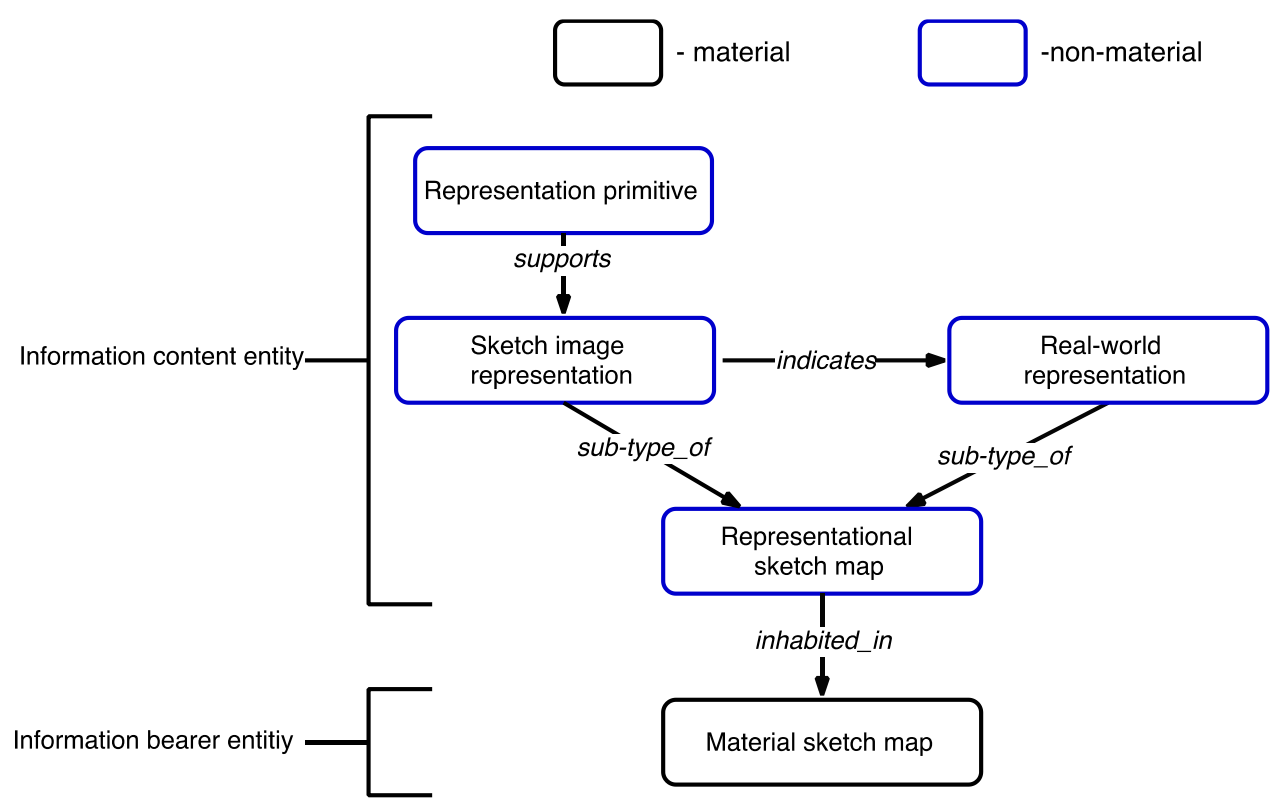

Figure 3. The hierarchy of sketch interpretation

\subsection{Material sketch map level}

At the material level, a sketch map is made of a collection of strokes, each created from pen down to pen up events. The locations and widths of strokes differ in online and offline sketching (Davis 2007). In online sketching with pen-based input devices, strokes are composed of time-stamped points, each of which has a position specified by coordinates $(\mathrm{x}, \mathrm{y})$. In offline sketch maps, strokes are a collection of marks written or drawn by pens on paper. The offline sketch maps must be scanned first as digital images and then pre-processed to enhance image qualities for further interpretation. The preprocessing takes into account both the imprecision of freehand drawings such as overlaps, gaps and wiggles, and typical image effects such as inhomogeneous illumination (Davis 2007, Broelemann et al. 2011). We only consider offline sketch maps in the current study.

We reuse the existing taxonomy created by Galton and Worboys (2011) to create our ontology at the material sketch map level (Figure 4). The ontology in the figure has the twofold division defined in BFO (continuants $v s$. occurrents) as the toplevel distinction between entities. The sketch information entity is without any material part and has the physical sketch map as its bearer. We include the sketch information entity here to show the link between the material level and the non-material representational level. The details of the information entity will be elaborated in the next section. 


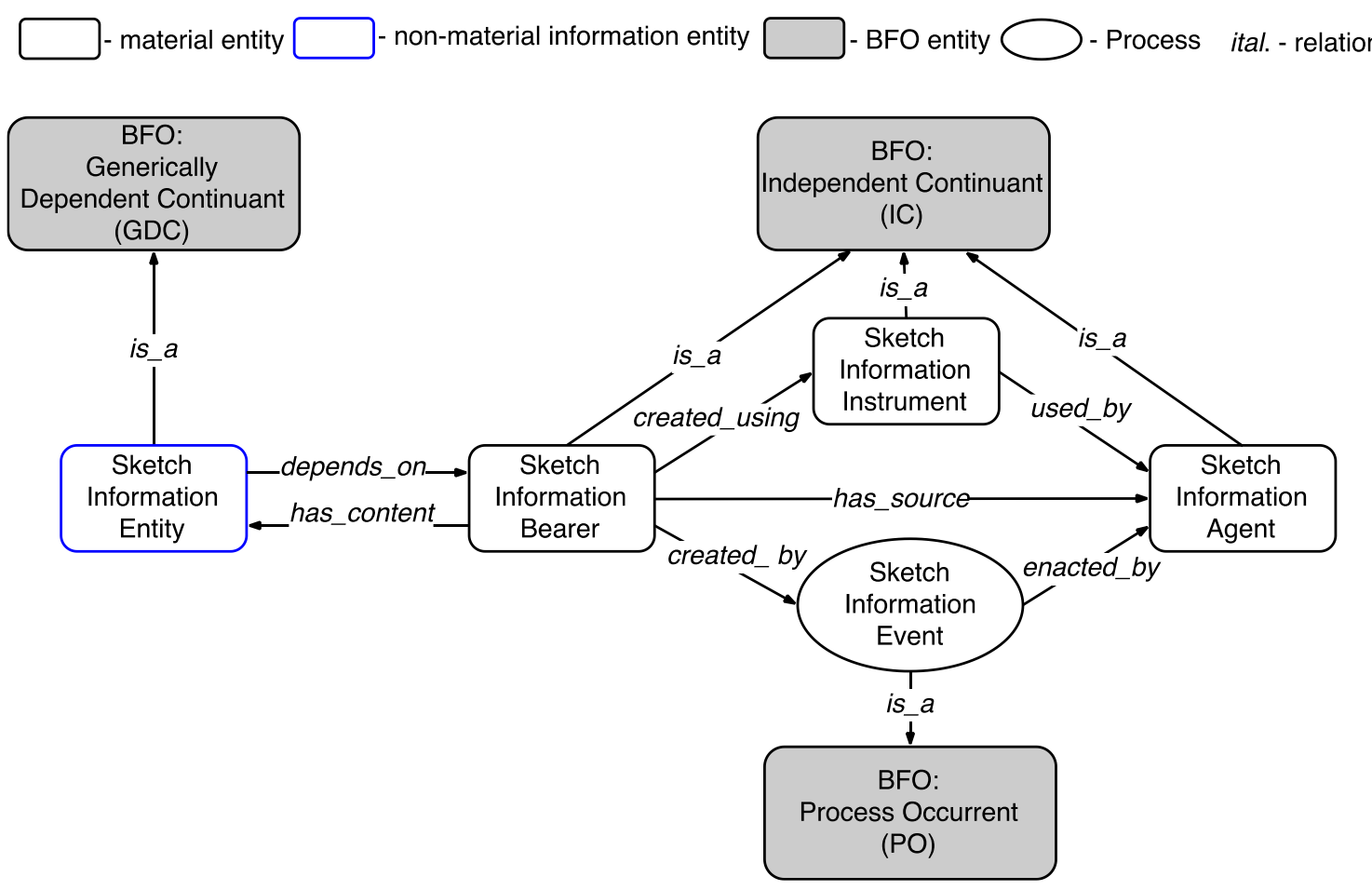

Figure 4. Sketch map ontology based on Galton and Worboys (2011)

In Figure 4, all the entities except the sketch information event are continuants that persist through time. The sketch information event is a process occurrent that unfolds in time. The sketch information entity is a generically dependent continuant that is dependent on one or more independent continuants that can serve as its bearers. It can be either explicitly encoded or implicitly supported by the physical sketch map as its information bearer. In this paper, the sketch information bearers are paper-based offline sketch maps. These sketch maps are created by sketch information events carried out by sketch information agents as sketch map producers. Galton and Worboys (2011) defined two types of information event: the information origination event and information copying event. In our case, the sketch information entity is originally generated by drawing on paper as an information origination event, and the subsequent propagation of the information in a succession of new bearers is accomplished by a series of information copying events such as photocopying and scanning.

\subsection{Representational sketch map level}

The physical strokes are not understandable to a human being without further structural and semantic data to interpret them. Such interpretations are performed at the nonmaterial, representational sketch map level embedded in the strokes as their bearers. At this level, sketch image and real-world representations supported by representation primitives convert raw strokes into meaningful sketch information. The representational sketch elements and the hierarchy within this level are described below. Figure 5 shows the overall structure of the level.

\subsubsection{Representation primitives}

The bottom-level representation primitives support the image and real-world representations at higher levels. Following the convention of text/graphics separation in document analysis (Tombre et al. 2002), we distinguish two types of primitive elements 
as textual and graphic. Both types of elements are further recognised at two sub-levels, namely grapheme and morpheme. Note that the approach to find primitive elements is out of our research scope. Existing methods from sketch recognition research can be found from the image recognition domain literature (e.g., Sezgin et al. 2006).

The text in our sketch maps usually comprises written words which are used for annotating or labelling graphic objects. The primitive textual elements are letters in words. We called these letters graphemes as they are the smallest units of writing. Words are composed of letters and exist at the morphemic level. We borrow the term morpheme from linguistics to denote words as the smallest meaningful textual elements in sketch interpretation. Note the difference in defining morpheme in linguistics, where morphemes can also be parts of words (Catford 1965).

Using the same categorisation, graphical objects can also be assigned to graphemic and morphemic levels. Graphic elements at the graphemic level such as straight lines and arcs are the smallest indivisible graphic units of sketching. Basic shapes such as individual blobs and squares, formed using these straight lines and arcs, are recognised at the morphemic level. The relationships of these graphic primitives must obey a set of geometric constraints such as parallelism and orthogonality (subject to tolerance) to be instances of particular shapes. Due to physical sketching imprecision, tolerance is necessary in defining the geometric constraints. For example, it has been found that orthogonality in sketch maps is usefully defined as the relationship of two lines at right angles $\left(90^{\circ} \pm 5^{\circ}\right)$ (Wang 2014). Two other methods of shape recognition can also be applied here (Davis 2007): the first considers the sequence of the graphical primitives used (if available), i.e., how the shape is drawn; and the second considers the traditional concept of image appearance, i.e., what the shape looks like. Shapes recognised at this level are mostly done by using the classic isolated recognition method (Broelemann et al. 2016). 


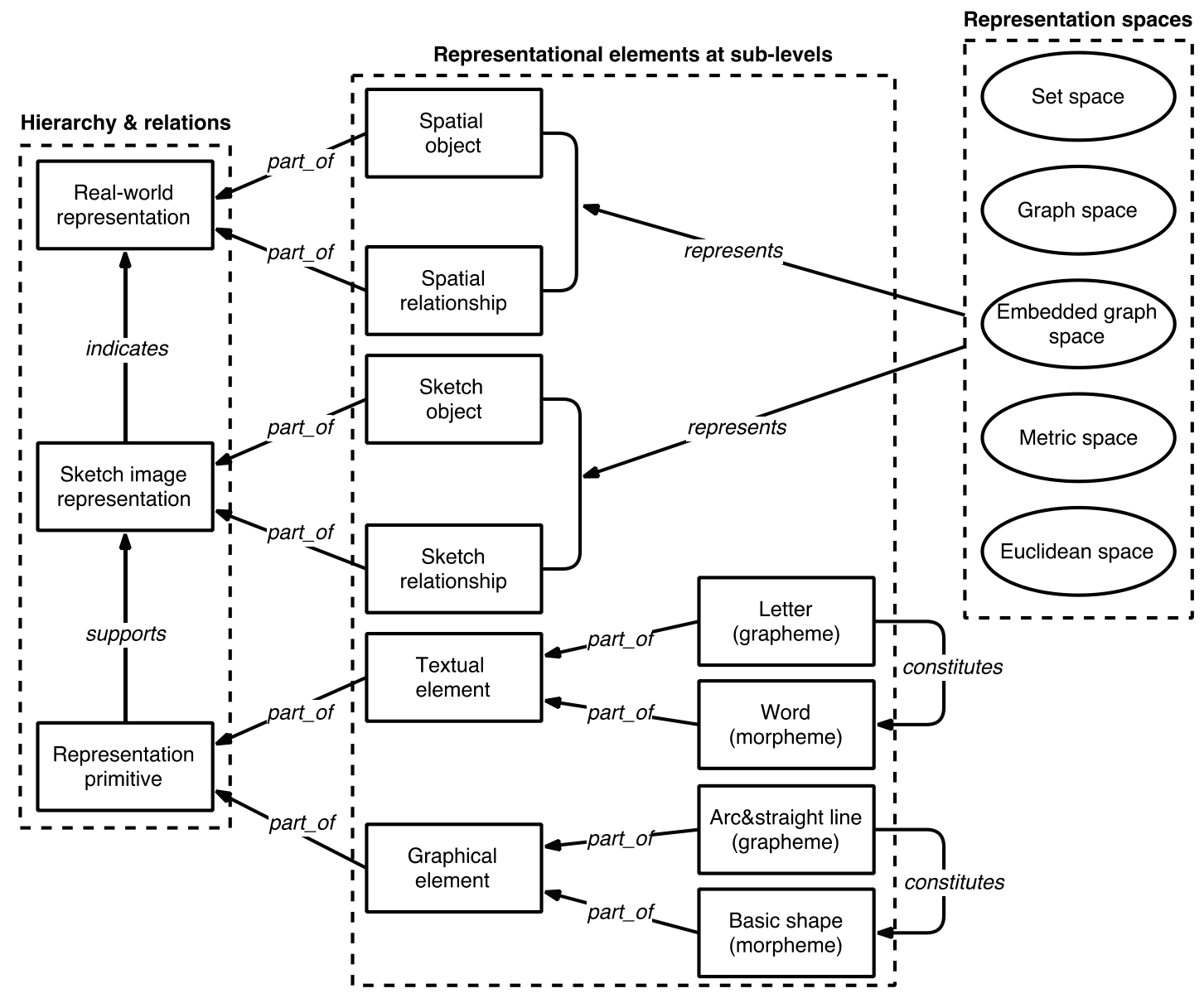

Figure 5. Structure and categorisation of the elements at the representational sketch map level

\subsubsection{Sketch image representation}

Supported by the basic geometric shapes labelled by words, a sketch image representation addresses only the visual features, particularly geometry, of a sketch image without making use of any semantics about the depicted reality. This representation is used for the literal interpretation of a sketch image. This bears resemblance to the composition in the visual arts in that it only deals with the placement of visual elements such as lines and shapes but not the subject of a work.

Elements at this level are a collection of sketch objects formed by using representation primitives, and the sketch relationships between these objects. A sketch object is defined as a user-drawn object consisting of basic geometric shapes and optionally labelled by words. The labels represent what shapes denote. A sketch object is similar to a glyph defined in CogSketch (Forbus et al. 2011), where each glyph represents a user-drawn object composed of ink (corresponds to the arcs and straight lines as graphemic graphical primitives) and content (corresponds to the words as morphemic textual primitives). Shapes of sketch objects can be 0-D points, 1-D straight lines and curved arcs, and simple 2-D polygons, and they can also be higher-order complex shapes composed of simple shapes of mixed dimensions. Optionally, these shapes can be labelled with one or more words inside or next to them. Figure 6a shows a raw sketch map and Figure $6 \mathrm{~b}$ shows its processed sketch image with basic shapes and words recognised. In the processed image, examples of sketch objects are the areal object that has the word 'Bus' inside, and the linear objects forming 'Oxford Street'. 
Sketch objects with higher-order shapes are circled in red in the figure. The cross and square shapes form a new shape, which looks like a church symbol. The intersected line and circle form the symbol of a tube station used in London.

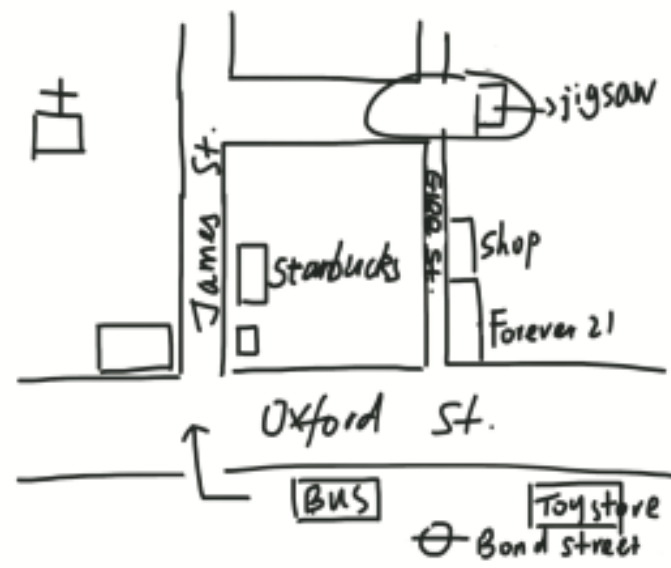

(a) raw sketch map

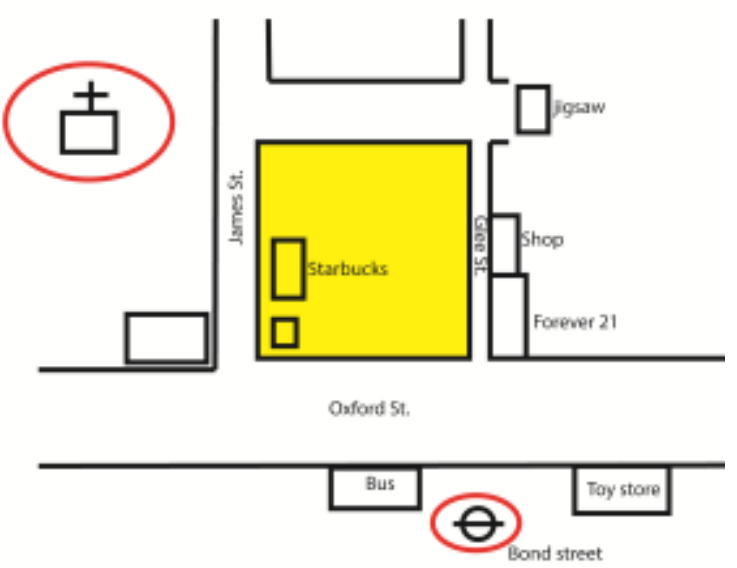

(b) processed sketch image

Figure 6. An example of sketch image representation

Sketch relationships are visual relationships calculated between sketch objects, and different relationships can be calculated depending on the representation space they use (more details in the next paragraph). Such relationships allow us to resolve ambiguity between sketch objects with similar appearance so they are important for sketch map interpretation. Sketch relationships between sketch objects lead to inferences of conceptual relationships of real-world objects being sketched. For example, the sketch relationship 'contains' indicates a conceptual containment relation between real world objects, café (Starbucks) and street block (highlighted in Figure 6 (right)).

We now consider using the following five formal spaces of sketch objects, which represent and reason about a sketch image at different levels. These spaces have been chosen because they provide a tiered formal representation based on standard mathematical structures. For each space, we include an example in Figure 7 to show how it can be used for sketch representation.

Set space. A set of sketch objects provide the simplest representation of a sketch image. When a sketch image is defined as a set, the constituent sketch objects are called members of the set. A sketch image can be represented either as comprising three subsets $S_{\text {point }}, S_{\text {linear }}$ and $S_{\text {areal }}$ to classify sketch objects of different dimensionality, or as two subsets $P_{\text {label }}$ and $P_{\text {nolable }}$ to distinguish sketch objects with or without labelling. Table 1 gives an example of the types of sketch objects classified regarding their geometric shapes in a plane.

The set space allows identification and classification of sketch objects. The remaining four spaces allow determination of spatial relationships between sketch objects. 
Table 1. Types of sketch objects in a set space

\begin{tabular}{|l|l|l|l|l|}
\hline Dimensionality & Major type & \multicolumn{2}{|l|}{ Sub-types } \\
\hline 0-D & Point objects & $\begin{array}{l}\text { Single point } \\
\text { Multipoint }\end{array}$ \\
\hline 1-D & Line objects & $\begin{array}{l}\text { Open line } \\
\text { Closed line }\end{array}$ & $\begin{array}{l}\text { Straight line } \\
\text { Curved line }\end{array}$ & $\begin{array}{l}\text { Single line } \\
\text { Multiline }\end{array}$ \\
\hline 2-D & Area objects & $\begin{array}{l}\text { Single polygon } \\
\text { Multipolygon }\end{array}$ \\
\hline
\end{tabular}

Abstract graph space. A sketch image is represented in an abstract graph space as a set of nodes (sketch objects) connected by a set of edges (connectedness as sketch relations). In practice, a sketch image needs to be processed first to have line segments (as edges) detected using the thinning algorithm (Guo and Hall 1989). Using the abstract graph structure, we can represent how sketch objects are connected to each other.

Embedded planar graph space. A further level of information may be added to the graph-based representation by embedding the graph in a 2-D Euclidean space, so that the structure of the graph is preserved. Edges are embedded as arcs that may only be intersected at nodes of a graph. This concept of embedding allows us to recognise different connectivities represented as inequivalent planar embeddings, even though they may be isomorphic in the abstract graph space. Since the concept of 'face' (flat planar surfaces) becomes available when a graph is embedded in a plane, we can also represent topological relations such as insideness, intersection and adjacency between sketch objects. Depending on the geometries of the sketch objects (Table 1), different topological relationships can be incorporated. For example, using the dimensionally extended 9-intersection model (DE-9IM), we can represent the relationships: equality, disjointness, intersection, touching, overlap, crossing, within, and containment between pairwise sketch objects of varied dimensionalities (Clementini and Di Felice 1996).

An important topological construct is that of 'boundary'. In the real world, both fiat and bona fide boundaries are found (e.g., county borders and river banks, respectively). Fiat boundaries separate fiat objects from their surroundings and enable a clear distinction of what does and does not belong to the objects (Kulik 2001). Different from bona fide objects, or genuine objects, fiat boundaries do not support the open/closed distinction on which the classic point-based topology is based (Smith and Varzi 2000). Our representation makes no distinction between these types, but this is scope for future work. Note that sketch objects (also spatial objects introduced in 3.2.3) are fiat objects and their boundaries are dependent on the cognitive and drawing processes.

Many spatial problems have been modelled using abstract or embedded planar graphs, where these problems became essentially the task of finding a suitable route through a network. The abstract and planar graphs form the network space introduced in Worboys and Duckham (2004), which is important to represent and reason about street networks in real world.

Metric space. In this space, the concept of distance is available, which we can use to calculate distances between point-like sketch objects. The concept of distance is necessary for defining qualitative positional relations between sketch objects such as at, nearby, in the vicinity, and far away, so that the concepts of neighbourhood as well as 
linear order relations based upon positional relations become available. Note that the topological relations can also be derived and represented in this space.

Euclidean space. In this space, the concepts of both distance and direction are available. As a highly organised kind of space, a Euclidean space brings richer geometries and several well-defined relationships that can act upon sketch objects of different dimensions, e.g., area-area directional relations and area-line distance relations. Both qualitative and quantitative spatial relationships such as topology, orientation and distance between sketch objects can be represented and computed in this space. The set-, graph- and metric-based spaces provide underlying structures for the Euclidean space.

\subsubsection{Real-world representation}

The real-world representation deals with the depicted real-world objects by using domain-specific knowledge. We discuss at this level the entities that are part of a sketched scene memorised from reality. This level arises from the previous representational sketch image level by further grouping or segmenting geometric shapes based on their visual sketch relationships and then matching them against labels or existing training samples and templates. Shape recognition at this level can take advantage of using the local context-aware recognition approach introduced by Broelemann et al. (2016), where we use the context given by surrounding sketch objects to get richer descriptions than the isolated recognition of independent shapes performed at the sketch image representation level. By using this approach, Broelemann et al. (2016) were able to recognise streets, city blocks (surrounded by streets and resembled by image background) and landmarks of different types such as parking lots and buildings. We can also infer various spatial relationships from the visual relationships available at each of the formal spaces together with some other information provided by sketch maps. For example, two adjacent sketch objects in the planar graph space might suggest that the depicted spatial objects are adjacent in reality (see the two adjacent shops located along Glee Street in Figure 6).

We define two types of real-world representational elements, namely spatial object and spatial relationship. Spatial objects represent artificial or natural entities in reality such as parks, streets, shops and canals. These objects are the cognitive reference points (Tversky 2000) externalised as sketches due to their perceptual salience, functional significance in the physical environment or their relevance to sketch tasks (Wang 2014). We can classify spatial objects, either as the five city elements from Lynch (1960) focusing on functionality, or as the four elements proposed by Wang (2014) focusing on spatial configurations. Spatial relationships are calculated between spatial objects and reflect in the human mind how spatial objects are located in space. Note that such relationships are not always true about the real world due to distortions and schematisations from cognitive errors.

The proposed five formal spaces can also be used here to interpret and reason about sketch maps at the real-world representation level (Figure 7). In the set space, the upper-left sketch map is modelled as a collection of three sets ( $\mathbf{S}_{\text {landmark }}, \mathbf{S}_{\text {street }}, \mathbf{S}_{\text {cityblock }}$ ), providing the types of spatial objects extracted from the map. For example, the four connected streets Glee, Oxford and James form the city block Oxford-Glee-James as the

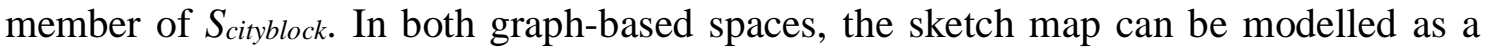
network of walking paths connecting street junctions as nodes, which reflects the sketch map creator's knowledge of location accessibility. The topological relations of 
insideness and adjacency are available in the embedded graph space. For example, Starbucks is inside the region bounded by Oxford, James and Glee streets, and an unknown building is adjacent to both Oxford and James street. Having the concept of distance available in the metric space, the size of each spatial object can be computed and compared, so we can draw certain conclusions such as "Oxford Street is wider than James Street and Glee Street is quite narrow". The concepts of linear ordering and neighbourhood are both available based on distances. Sketch maps usually do not have a single consistent global reference frame, and the concept of neighbourhood is necessary for representing local spatial relations (Meilinger 2008, Wang and Schwering 2015). Having neighbouring buildings with respect to the reference routes (red and blue routes highlighted in Figure 7), the linear order of spatial objects such as buildings and public transport stations located along the routes can be computed. Spatial objects, such as the toy store and church, which are not within the vicinity of the reference routes are excluded. In the Euclidean space, the concept of orientation becomes available. Having Glee Street as an oriented reference object, we can calculate directional relations using the method introduced in Wang and Schwering (2015), e.g., the shop Forever 21 is located at the back_right zone with respect to Glee Street. Having the junction of Oxford and James streets as the reference object, we can calculate the cyclic order of neighbouring spatial objects with respect to the junction. 
Sketch map

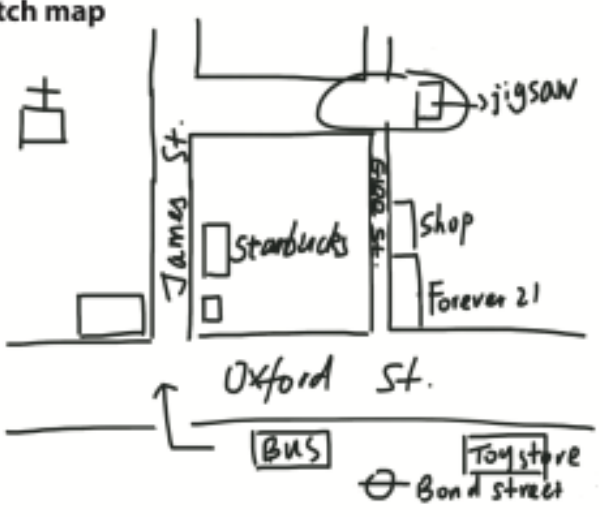

Embedded graph space
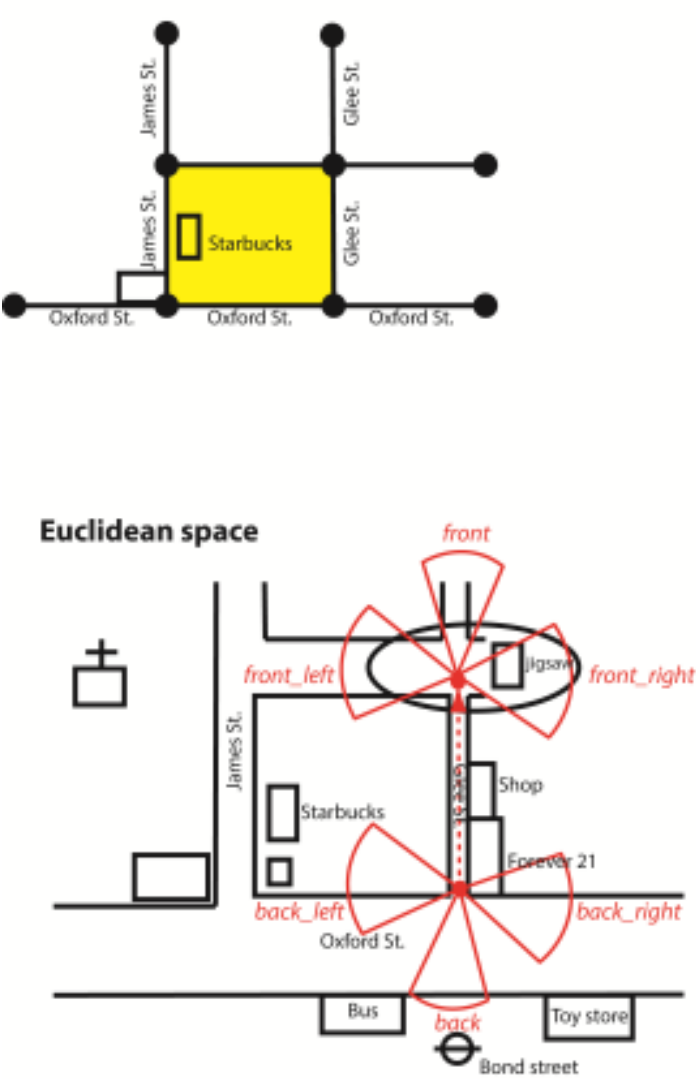
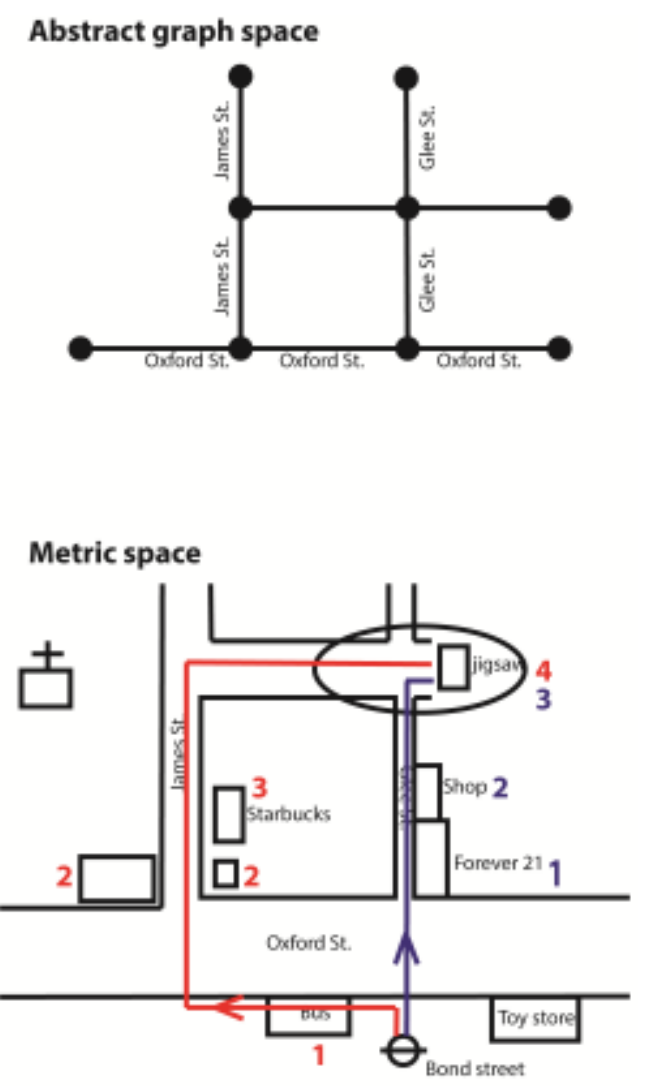

Euclidean space

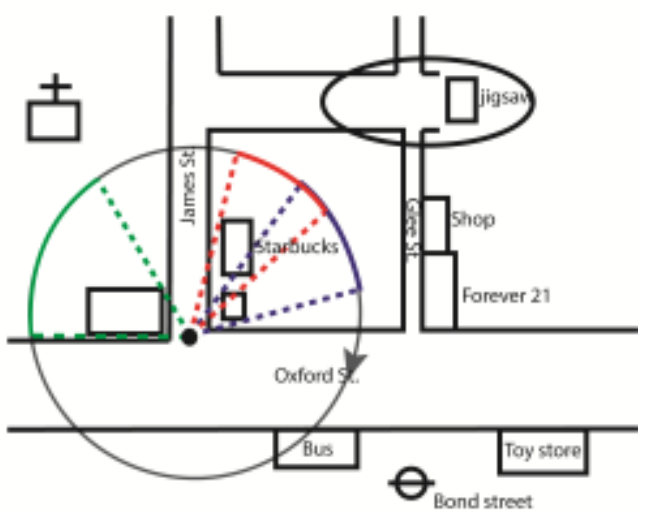

Figure 7. An example of real-world representations using different formal spaces

\section{Representational Sketch Maps in the IAO Framework}

The levels and spaces introduced above help to learn about the sketch information extracted from sketch maps. Based on Arp et al. (2015), sketch information as a generically dependent entity can exist in multiple copies and inhere in multiple information bearers including the original paper copy. These bearers all share the same pieces of information, in other words the same abstract pattern (Galton et al. 2016). Examples of abstract patterns are a collection of words composed of basic letters denoting a user-drawn object, or a collection of junctions connected by street segments denoting a street network. We introduce in this section a set of IAO-based ontologies of sketch information entities taking into account both the levels and the five formal representation spaces. As information content is of the central interest in our domain, 
our ontologies focus only on the Information Content Entity (ICE), with each ontology representing the representational sketch map level introduced in section 3.2 (including representation primitive, sketch image representation, and real-world representation).

Figure 8 shows the ontology of a representation primitive built at the lowest level of representational sketch map. We reuse the five ICE entities (in blue) (image, textual entity, data item, Cartesian spatial coordinate datum and measurement datum). The raster sketch image in Figure 8 is a sub-type of IAO image made up of pixels, which comprises representation primitives containing graphic and textual components. Straight lines and arcs are graphic entities comprising basic geometric shapes. Words of letters are textual entities used for denoting shapes and describing sketches. Each representation primitive is composed of pixels, each of which has position and colour defined using the ICE entities, spatial coordinate datum and measurement datum, respectively. Details of the reused ICE entities can be found in IAO (2014).

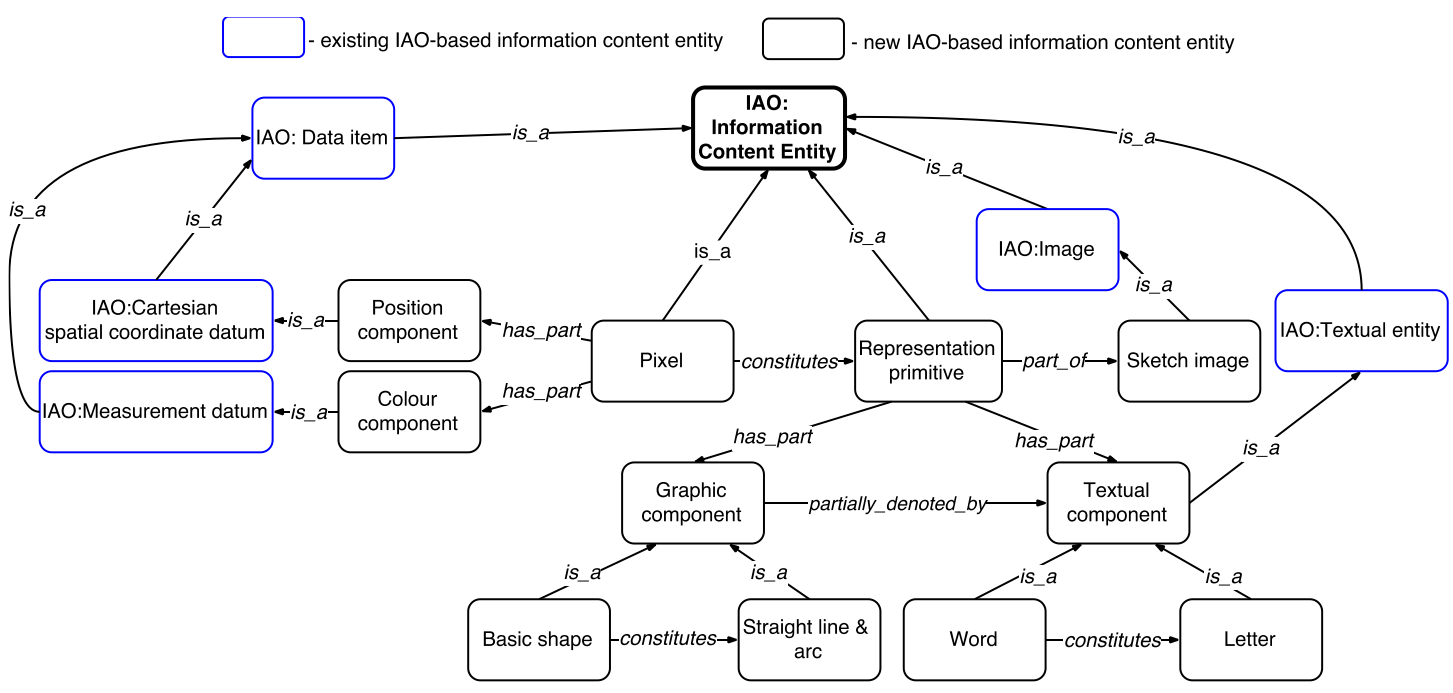

Figure 8. An ontology of sketch image primitives based on IAO

Figure 9a illustrates an ontology of the higher level sketch image representation using the five formal spaces. The vectorised sketch image is now conceived as made up not of primitives but of sketch objects. Different types of sketch relationships between sketch objects are made available based on the choice of the formal space. These sketch relationships lead to inferences of conceptual relationships of real-world objects. Figure $9 \mathrm{~b}$ provides an example of an ontology of topological relationships of sketch objects using the planar graph space.

Similarly, using the five formal spaces, an ontology of the highest level realworld representation is shown in Figure 10a. Having the domain knowledge of what can be the real-world objects being depicted, the sketch image at this level is made up of spatial objects that are capable of multiple realisations in different physical forms in reality $^{2}$. These spatial objects are associated with the lower level by image segmentation and grouping of sketch objects based on their sketch relationships. Entities at this level are still information artefacts defined in such a way as to enable us to understand the underlying reality by reasoning about the formal spaces that they constitute. Figure $10 \mathrm{~b}$

\footnotetext{
${ }^{2}$ Multiple realisations can arise from the difficulty of aligning depicted spatial objects from a sketch map and spatial objects in reality.
} 
provides an example of an ontology of distance relationships of spatial objects using the metric space.

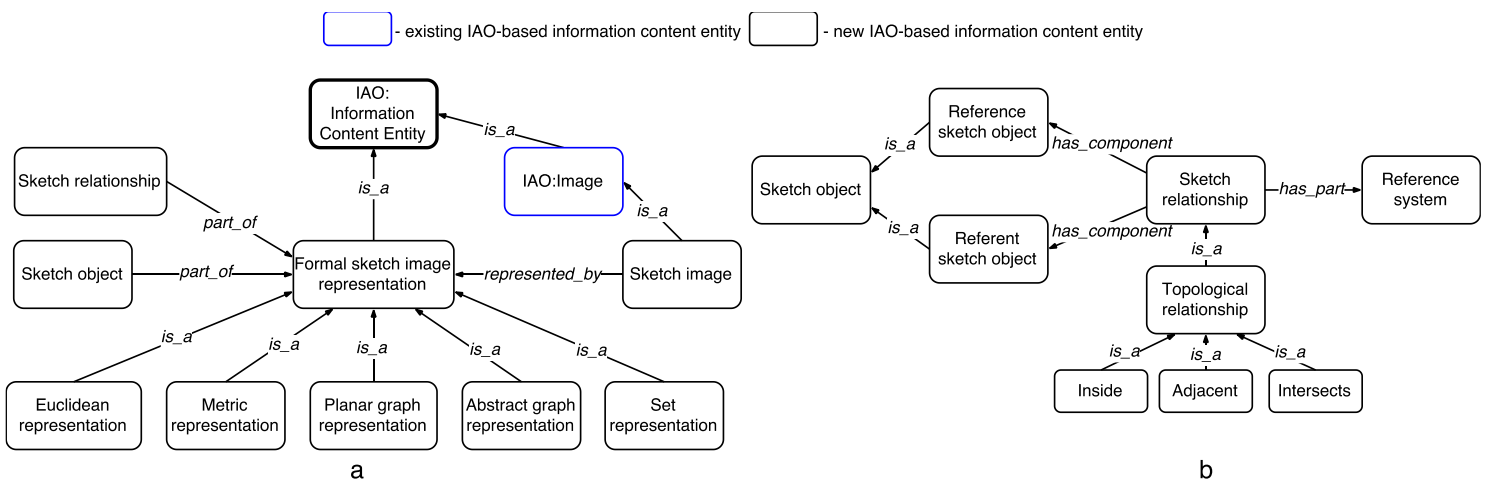

Figure 9. Generic ontological view of formal sketch image representation (a) and ontology of topological relationships of sketch objects (b)

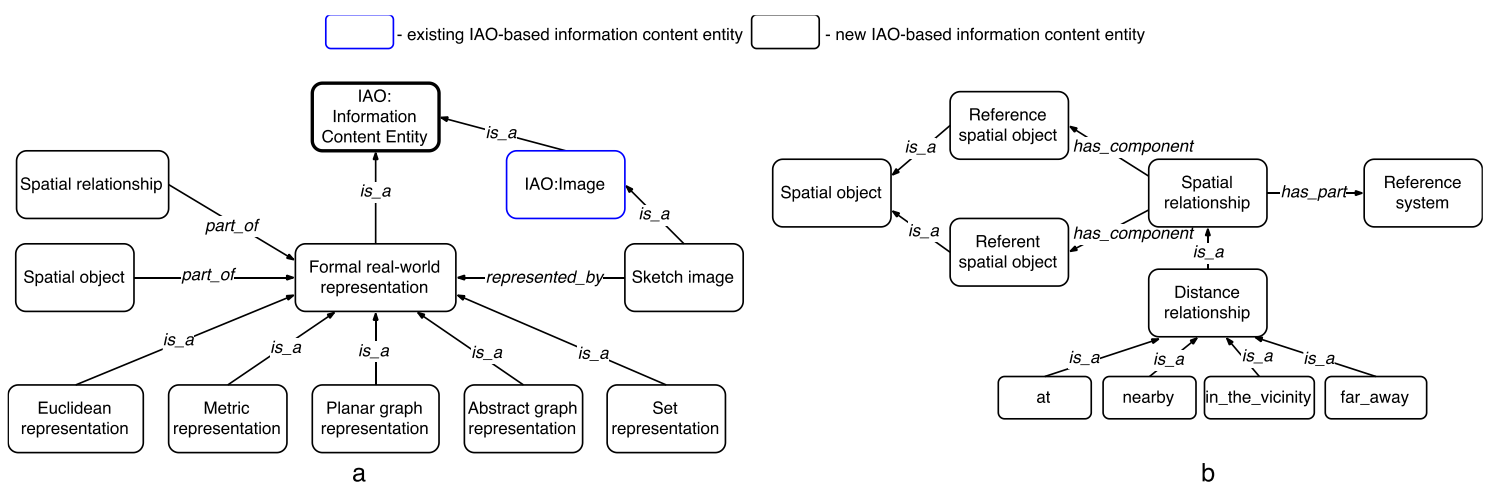

Figure 10. Generic ontological view of formal real-world representation (a) and ontology of distance relationships of spatial objects (b)

Similar to Hudelot et al. (2008), description logic can be used to formalise the representations of sketch/spatial relations, in order to clarify our understanding of them and to automate spatial reasoning. If a relation is binary, its inverse relation as well as its properties such as symmetry, asymmetry, reflexivity, and irreflexivity can be specified. Also, such a formalisation can help constrain the given relations. For example, in Figures $9 \mathrm{~b}$ and 10b, the relationships are not always interchangeable, i.e., order matters. If $x$ is adjacent to $y$, then $y$ is also adjacent to $x$, but if $x$ is inside $y$, then $y$ is not inside $x$.

\section{An Illustrative Example}

We present in this section an example of sketch map interpretation using the proposed method. We show how a raw sketch map can be analysed from the material to nonmaterial level, and from the literal sketch image level to the real-world level with richer semantics.

\subsection{Pre-processing physical sketch map}

Figure 11a shows a scanned version of an offline physical drawing made on paper. It is a raster online image consisting of pixels. This online drawing is then processed by binarisation for enhanced image quality and text/graphics separation for further graphic 
analysis. The binarisation method deals with monochromatic sketch maps by labelling each pixel either as background or as drawing. The graphic elements are further processed by region-based segmentation, and then object and shape recognition. We refer the reader to the work of Broelemann et al. (2016) for more details on the methods used in graphics recognition. Vectorisation, such as imaging tracing is also necessary before carrying out the formal representations at different levels.

\subsection{Sketch image primitives}

Figures $11 \mathrm{~b}$ and $11 \mathrm{c}$ illustrate the ideas of text/graphic separation and basic representation primitives. Open and closed arcs in Figure 11b are the basic graphemic graphic entities, which are further processed to become instances of basic geometric shapes using constraints such as parallelism and orthogonality. (For the purposes of this paper, this figure is made manually. In practice, the figure is usually generated by a series of graphics recognition methods such as region-based segmentation. Graphic features are then identified by the boundaries of recognised regions.) These geometric constraints, as discussed before, are subject to the tolerance used by certain image analysis algorithms because of the imprecision from freehand sketches. We highlight in red the minimum bounding boxes as the approximate shapes of the graphic primitives. Note that some of the incomplete open shapes (usually found at the edge of the paper) need to be completed. In Figure 11c, the smallest disjoint units are letters which form words as the smallest meaningful units for denotational purpose.

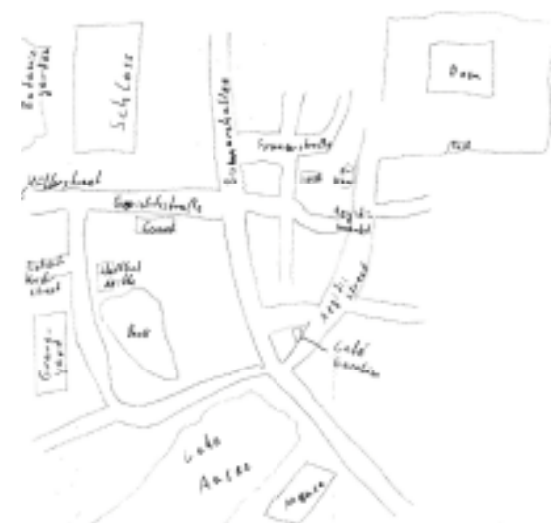

(a)

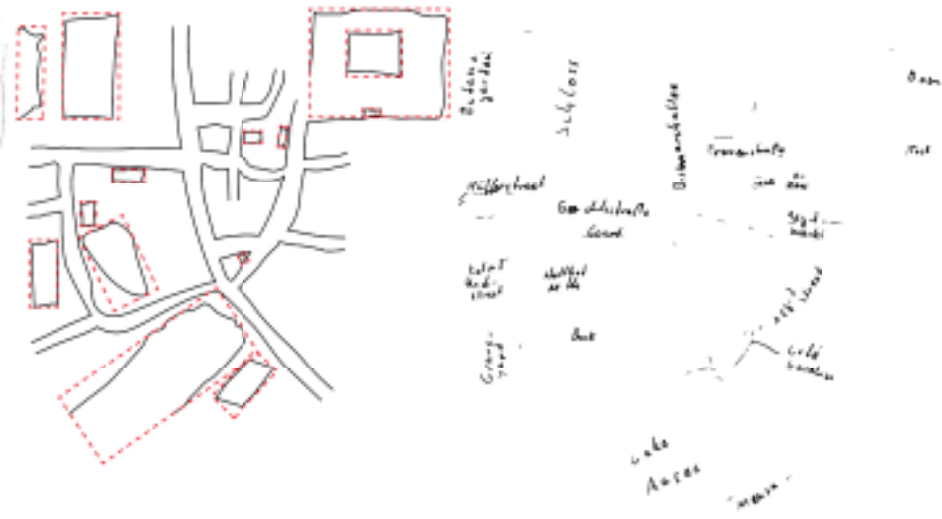

(b)

(c)

Figure 11. (a) scanned offline sketch map of downtown Muenster in Germany (b) graphic entities (c) textual entities

\section{$5.3 \quad$ Sketch image representation using formal representation spaces}

The sketch image supported by the graphic primitives consists of a set of sketch objects identified as different geometric shapes. The sketch is constructed in the set space as the combination of three sets of sketch objects, which are the areal objects (brown), the closed arcs (green) and the open arcs (blue) (Figure 12a). The visual sketch relationships of these identified sketch objects are represented by using the graph-, metric- and Euclidean-based formal spaces (Figures 12b to 12e). 

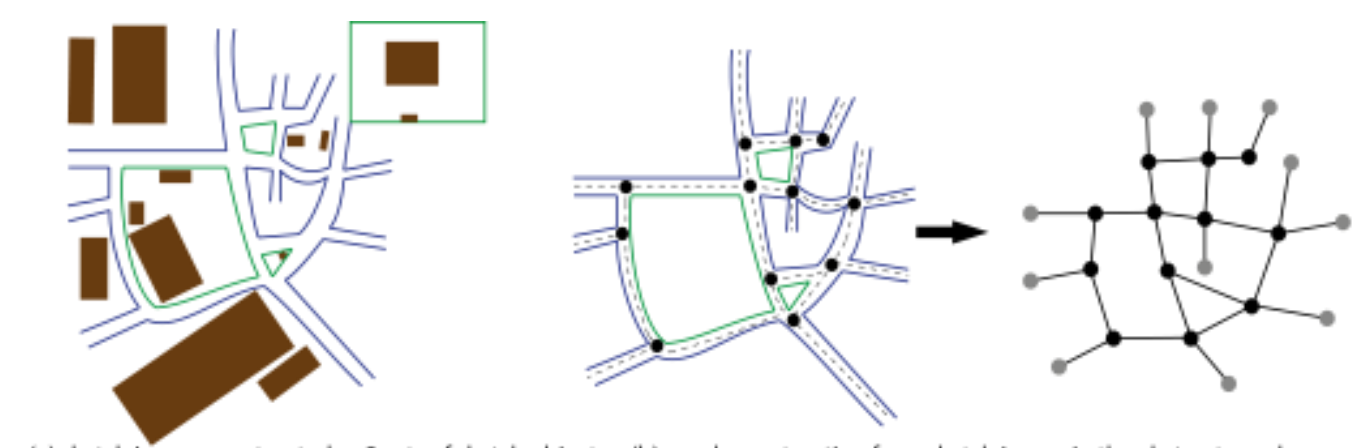

(a) sketch image constructed as 3 sets of sketch objects (b) graph construction from sketch image in the abstract graph space

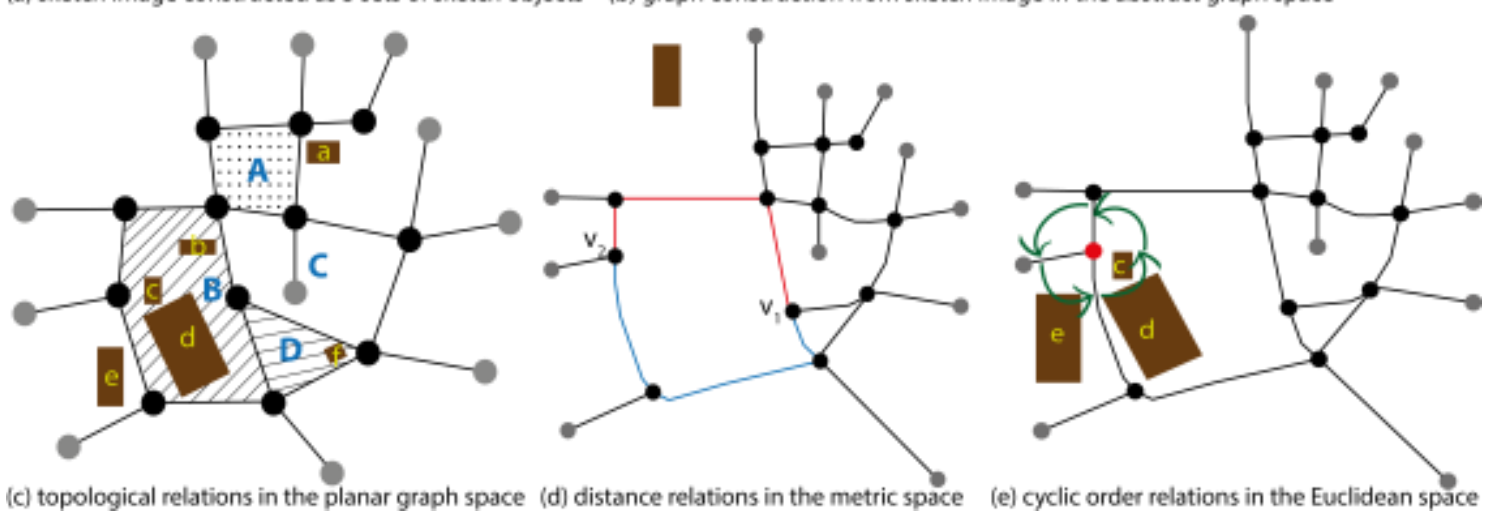

Figure 12. Sketch image representation using the five formal spaces

In the abstract graph space, the connectivity of sketch objects is made available. Figure $12 \mathrm{~b}$ illustrates the idea of the abstract graph construction: line segments as edges are detected from the image by the thinning algorithm. Edges are connected if they share the same node. We use grey to indicate those nodes that are connected to edges detected at the boundary of a sketch image. Figure 12c gives examples of topological relationships made available when an abstract graph is embedded in a 2-D plane. We use capital letters to differentiate the polygons created as the image background surrounded by closed line segments (A, B, C and D), and use different fill styles to denote these background polygons. Polygons share boundaries such as $\operatorname{touch}(B, D)$ and $\operatorname{touch}(C, D)$. A polygon can be inside another one such as inside $(f, D)$ and inside $(d, B)$. The metric space allows us to differentiate distances between nodes along different paths. For example, the distances between $v_{1}$ and $v_{2}$ along the two shortest paths (blue and red) are different (Figure 12d). A 2-D Euclidean space also brings in the concept of orientation, and all the sketch relations from the previous spaces are available in this space. Figure 12e shows the cyclic order relation between areal sketch objects with respect to a node $(\operatorname{cyclic}(e, d, c))$.

\subsection{Real-world representation using formal representation spaces}

The real-world representation includes sketchers' mental concepts of depicted realworld objects. Figure 13 shows the real-world representation of the sketch map described in section 5.1. Figure 13a shows a set of spatial objects. These include artificial buildings such as the café and library, natural geographic objects such as the lake, and the spatial objects that people usually share various opinions about their boundaries. The dotted-lines represent such vaguely delineated objects. e.g., the square where the cathedral is located was drawn as the Dom Plaza (Figure 13a). The other spatial relations between real-world objects shown in the figure mirror the spatial 
relations between sketch objects that are already discussed in Figures $13 \mathrm{~b}$ to $13 \mathrm{e}$. Different fill styles in Figure 13c denote different polygons, A, B, C and D, created as the image background surrounded by closed line segments. In Figure 13f, the neighbourhood of the reference street junction (in red) is decided using Voronoi cells based on distances between points (Figure 13e).

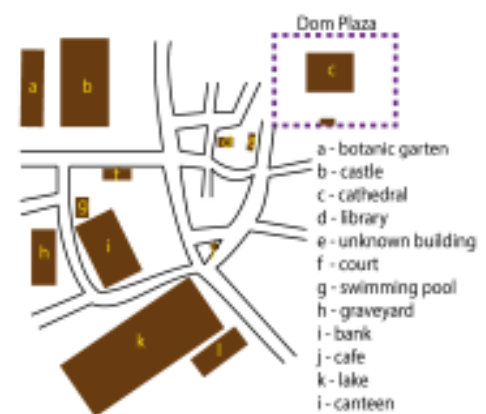

(a) sketch image constructed as recognised spatial objects (b) abstract graph of street network

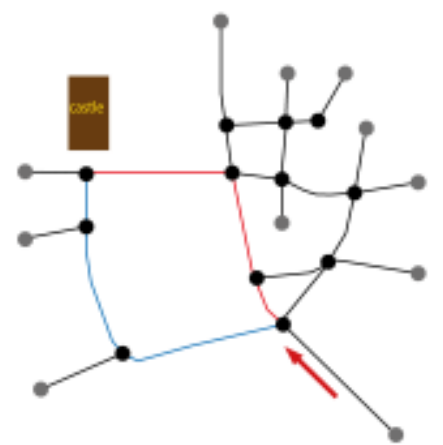

(d) distance relations in the metric space
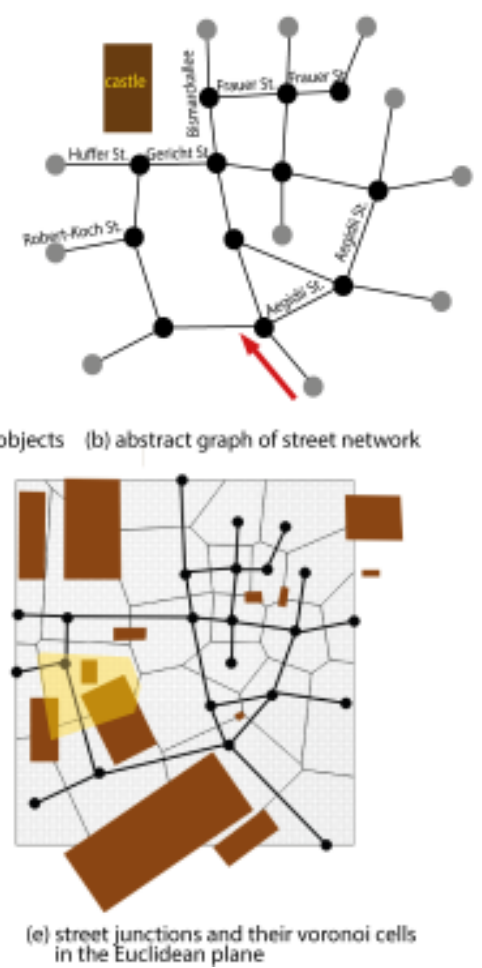

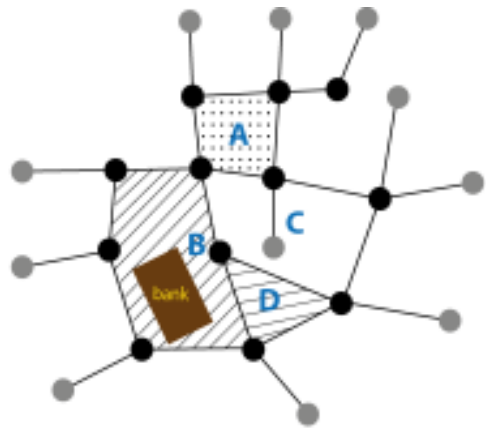

(c) topological relations in the planar graph space

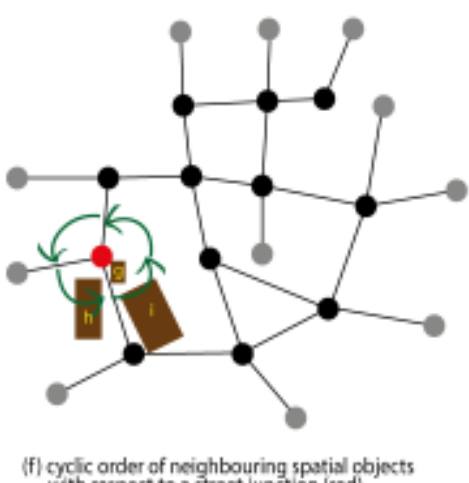

cyclic order of neighbouring spatial objects

Figure 13. Real-world representation using the five formal spaces

\section{Conclusions and Future Work}

We have advocated the adoption of an ontological point of view to obtain a clear sketch map interpretation and representation. In particular, we have developed a well-defined hierarchical structure to which the various representational entities handled during sketch interpretation can be assigned, and proposed the use of five formal representation spaces to classify and qualify spatial features and analyse their relationships at different levels.

The ontological contribution can help to establish a common interpretation of sketch maps by linking distinctive sketch entities defined at different levels, from the lowest material levels to the highest real-world representational level. The proposed ontologies also facilitate schema construction for sketch map databases, integration of sketch maps from different resources for knowledge sharing, and integration with the existing data from spatial databases for querying user-generated sketch content. For example, urban planners can use our methods to apply sketches as user-generated content to provide structured information on citizens' perceptions of their environment (e.g., SketchMapia proposed by Schwering et al. (2014)).

Formal representation spaces provide a systematic approach to sketch information extraction and spatial representation. This approach assists in the development of an automated system of spatial knowledge externalisation from sketch mapping. By externalising spatial knowledge, we may be able to provide new 
explanations of the following questions about cognitive mapping and human navigation: What is the common structure and type of spatial knowledge underlying human navigation that can be reflected on sketch maps? Is the structure similar to a graph (Chrastil and Warren 2014), or the structure rather reflects Meilinger's theory (2008) of network of reference frames? The identification, classification and qualification of spatial relationships including topology, orientation and distance can also help to understand people's sense of place, especially the structural aspects of places (e.g., Curtis et al. 2014).

The current study focuses on the interpretation of sketch maps associated with urban areas from a survey perspective. The ontologies we provide here are not complete. Their extension and population require the diversification of the range of sketch maps from different perspectives. For example, sketch maps from a route perspective, sketched spaces of different spatial scales (smaller indoor space or larger geographic space), and sketch map producers with varied cultural backgrounds. Other representations of space, such as fractal space, may also be incorporated in the proposed ontologies if fractal geometry and patterns can be found in other types of sketch maps. We shall also consider revising the proposed ontologies by integrating them with existing widely recognised suites of ontologies. The evaluation of the ontologies by users is also necessary, which will verify if the proposed ontological resources help to close the semantic gap between the perceptual level and conceptual level. Smeulders (2000, p. 1) defined such a gap as 'the lack of coincidence between the information that one can extract from the visual data (perceptual) and the interpretation that the same data have for a user in a given situation (conceptual)'.

The ultimate goal is to make sketch interpretation automatic. To do this, a set of rules needs to be defined to decide the level, representation space and type of sketch features that are required to be extracted in accordance with the sketch map type and application. Formalisation of ontologies is needed and will be our next step to clarify diverse understandings of relationships of sketch entities and to automate spatial reasoning. Cooperation with computer vision specialists is also necessary to develop such an automated system.

\section{Acknowledgements}

This work was partially funded from the University of Greenwich grant on "Smart Transport in a Smart City" and the AGILE (Association of Geographic Information Laboratories in Europe) grant for early career researchers.

\section{References}

Appleyard, D., 1970. Styles and methods of structuring a city. Environment and Behavior, 2(1), 100-117.

Arp, R., Smith, B., and Spear, A. D., 2015. Building Ontologies with Basic Formal ontology. Cambridge, Massachusetts: MIT Press.

Billinghurst, M., and Weghorst, S., 1995. The use of sketch maps to measure cognitive maps of virtual environments. In Proceedings of Virtual Reality Annual International Symposium, 11-15 March 1995, Research Triangle Park, North Carolina, USA: IEEE, 40-47.

Blades, M., 1990. The reliability of data collected from sketch maps. Journal of Environmental Psychology, 10(4), 327-339. 
Broelemann, K., Jiang, X., and Schwering, A., 2011. Automatic street graph construction in sketch maps. In International Workshop on Graph-Based Representations in Pattern Recognition, 18-20 May 2011, Muenster, Germany: Springer Berlin Heidelberg, 275-284.

Broelemann, K., Jiang, X., and Schwering, A., 2016. Automatic understanding of sketch maps using context-aware classification. Expert Systems with Applications, 45, 195-207.

Catford, J. C., 1965. A linguistic theory of translation. London: Oxford University Press.

Chen, J., and Takagi, N., 2013. A pattern recognition method for automating tactile graphics translation from hand-drawn maps. In 2013 IEEE International Conference on Systems, Man, and Cybernetics, 13-16 October 2013, Manchester, UK: IEEE, 4173-4178.

Chrastil, E. R., and Warren, W. H., 2014. From cognitive maps to cognitive graphs. PloS one, 9(11), e112544.

Clementini, E., and Di Felice, P., 1996. A model for representing topological relationships between complex geometric features in spatial databases. Information Sciences, 90(1), 121-136.

Curtis, J.W., et al., 2014. The prospects and problems of integrating sketch maps with geographic information systems to understand environmental perception: A case study of mapping youth fear in Los Angeles gang neighborhoods. Environment and Planning B: Planning and Design, 41(2), 251-271.

Davis, R., 2002. Sketch understanding in design: Overview of work at the MIT AI lab. In 2002 AAAI Spring Symposium, 25-27 March 2002, Palo Alto, California: AAAI, 24-31.

Davis, R., 2007. Magic paper: sketch-understanding research. IEEE Computer, 40(9), 34-41.

Forbus, K., et al., 2011. CogSketch: Sketch understanding for cognitive science research and for education. Topics in Cognitive Science 3(4), 648-666.

Galton, A., and Worboys, M., 2011. An ontology of information for emergency management. In Proceedings of the $8^{\text {th }}$ International Conference on Information Systems for Crisis Response and Management, 8-11 May 2011, Lisbon.

Galton, A., et al., 2016. Ontological levels in histological imaging. Frontiers in Artificial Intelligence and Applications, 283, 271-284.

Gangemi, A., et al., 2002. Sweetening ontologies with DOLCE. In Proceedings of the $13^{\text {th }}$ International Conference on Knowledge Engineering and Knowledge Management, 1-4 October 2002, Siguenza, Spain: Springer London, 166-181.

Guo, Z., and Hall, R. W., 1989. Parallel thinning with two-subiteration algorithms. Communications of the ACM, 32(3), 359-373.

Hudelot, C., Atif, J., and Bloch, I., 2008. Fuzzy spatial relation ontology for image interpretation. Fuzzy Sets and Systems, 159(15), 1929-1951.

IAO., 2014. Available from: http://www.ontobee.org/ontology/IAO?iri=http://purl.obolibrary.org/obo/IAO_0 000308 [Accessed 25 October 2016]

Ishikawa, T., et al., 2008. Wayfinding with a GPS-based mobile navigation system: A comparison with maps and direct experience. Journal of Environmental Psychology, 28(1), 74-82.

Kuipers, B., 1983. The cognitive map: Could it have been any other way? In: H. L. Pick Jr. and L. P. Acredolo, eds. Spatial Orientation - Thoery, Research, and Application. New York: Plenum Press, 345-359. 
Kulik, L., 2001. A geometric theory of vague boundaries based on supervaluation. In Proceedings of the $5^{\text {th }}$ International Conference on Spatial Information Theory, 19-23 September 2001, Morro Bay, CA, USA: Springer Berlin Heidelberg, 4459.

Lloyd, R., and Heivly, C., 1987. Systematic distortions in urban cognitive maps. Annals of the Association of American Geographers, 77(2), 191-207.

Lynch, K., 1960. The image of the city. Cambridge, Massachusetts: MIT Press.

Meilinger, T., 2008. The network of reference frames theory: A synthesis of graphs and cognitive maps. In Proceedings of the $6^{\text {th }}$ International Conference on Spatial Cognition, 15-19 September 2008, Freiburg Germany: Springer Berlin Heidelberg, 344-360.

Montello, D. R., 1993. Scale and multiple psychologies of space. In: A.U. Frank and I. Campari, eds. Spatial Information Theory: Theoretical Basis for Geographic Information Science. Lecture Notes in Computer Sciences No. 716, Berlin: Springer-Verlag, 312-321.

Newcombe, N., 1985. Methods for the study of spatial cognition. In: R. Cohen, ed. The development of spatial cognition. Hillsdale, NJ: Lawrence Erlbaum Associates, 277-300.

Niles, I., and Pease, A., 2001. Towards a standard upper ontology. In Proceedings of the $2^{\text {nd }}$ International Conference on Formal Ontology in Information Systems, 17-19 October 2001, Oqunquit, ME, USA: ACM, 2-9.

Schwering, A., et al., 2014. SketchMapia: Qualitative representations for the alignment of sketch and metric maps. Spatial Cognition \& Computation, 14(3), 220-254.

Sezgin, T. M., Stahovich, T., and Davis, R., 2006. Sketch based interfaces: early processing for sketch understanding. In Proceedings of the Special Interest Group on Computer Graphics and Interactive Techniques Conference, 30 July-3 August 2006, Boston, MA, USA: ACM, 22-22.

Smeulders, A. W., et al., 2000. Content-based image retrieval at the end of the early years. IEEE Transactions on Pattern Analysis and Machine Intelligence, 22(12), 1349-1380.

Smith, B., et al., 2013. IAO-Intel: an ontology of information artifacts in the intelligence domain. In Proceedings of the $8^{\text {th }}$ Conference on Semantic Technologies for Intelligence, Defense, and Security, 12-15 November 2013, Fairfax, VA, USA: CEUR, 33-40.

Smith, B., and Varzi, A.C., 2000. Fiat and bona fide boundaries. Philosophical and Phenomenological Research, 401-420.

Smith, B., and Malyuta, T., 2015. IAO-Core: Towards a general-purpose, top-level information artifact ontology. Available from: http://documents.tips/documents/iao-core-towards-a-general-purpose-top-levelinformation-artifact-ontology-5686638001112.html [Accessed 25 October 2016$]$

Tolman, E.C., 1948. Cognitive maps in rats and men. Psychological Review, 55(4), 189208.

Tombre, K., et al., 2002. Text/graphics separation revisited. In Proceedings of the $5^{\text {th }}$ International Workshop on Document Analysis Systems, 19-21 August 2002, Princeton, NJ, USA: Springer Berlin Heidelberg, 200-211.

Tversky, B., and Lee, P.U., 1999. Pictorial and verbal tools for conveying routes. Lecture Notes in Computer Science, 51-64.

Tversky, B., 2000. Levels and structure of spatial knowledge. Cognitive Mapping: Past, Present and Future, 24-43. 
Wang, J., 2014. Qualitative sketch aspects for sketch map alignment. Thesis (PhD). University of Muenster.

Wang, J., and Schwering, A., 2015. Invariant spatial information in sketch maps-a study of survey sketch maps of urban areas. Journal of Spatial Information Science, 2015(11), 31-52.

Worboys, M., and Duckham, M., 2004. GIS: A computing perspective. Boca Raton, FL: CRC press. 